\title{
A Two Parameter Fracture Mechanics Approach to Analyze Threaded Fasteners of A High Strength Steel
}

\author{
Clayton Reakes, Xiaosheng GAO and Srivatsan TS* \\ Department of Mechanical Engineering, the University of Akron, Akron, USA
}

Received: July 06, 2017; Accepted: August 23, 2017; Published: August 29, 2017

*Corresponding author: Srivatsan TS, Department of Mechanical Engineering, The University of Akron, Akron, Ohio 44325-3903, USA, E-mail: tsrivatsan@uakron.edu

\begin{abstract}
This paper presents a numerical investigation using the two-parameter fracture mechanics approach for studying threaded fastener geometries. It was found that the constraint arising from the presence of a surface crack in a threaded fastener does influence the stress in the forward sector. Consequently, the actual stress field shows a deviation from the theoretical HRR solution. Implementation of the J-Q methodology provided an approach for approximating the threaded fastener stress state using a two-dimensional modified boundary layer representation. Results reveal the parameter $Q$ to vary along the crack front and having lowest values at the free surface and peaking at the root of the crack. Lastly, it is shown that the magnitude of the parameter $Q$ is influenced by the magnitude of loading, thereby increasing the dominance of the large-strain region.
\end{abstract}

Keywords: Threaded fasteners; Cracks; Flaw size; Fracture mechanics; Finite element analysis; J-Integral; Q-Parameter;

\section{Introduction}

With the inevitability of surface flaws finding their way into mechanical components of all types by way of (i) damage to the surface, (ii) embrittlement caused by hydrogen and (iii) stress corrosion cracking, it is essential to have a full picture of the overall fracture mode at the time of failure for specific geometries. A most commonly fractured component in any design is the fasteners that see large separation forces during their operation. Case studies presented by Ramachandran [1] give many examples of fastener components failing during service. For these reasons, a much-needed interest in fracture properties for threaded fastener geometries with specific focus on nonlinear fracture mechanics has been developed.

In a previous study, it was found that the J-integral values of a crack front on a threaded fastener are highly reliant on geometric factors to include both flaw size and orientation [2]. In many situations for the case of complex components where low constraint geometry exists, the J-integral method begins to lose its validity once the region of large strain grows to a point where the HRR field can no longer be used to characterize the behavior within the plastic zone $[3,4,5]$. At this point, a multi-parameter approach becomes both necessary and essential to accurately represent the stress state, both at and near the tip of a crack. In this investigation, the J -Q approach [6] was used to examine its applicability for defining both the stress state and deformation state of flaws in fastener geometries.

\section{The Two-Parameter J-Q Theory}

The J-Q theory, introduced initially by O'Dowd and Shih [5,6,7] and investigated subsequently by Henry [8], relates directly to the presence of stress triaxiality in the geometry. In the J-Q theory, the J-integral is visualized to be representative of the deformation state at the crack-tip, with the addition of a $Q$ value to help define the stress characteristics prevalent at the tip of a flaw or crack. The $Q$ value is of interest since it parameterizes the stress field for distances ahead of the crack-tip within the J-Q annulus, normalized with respect to $J / \sigma_{\mathrm{y}}$. Many variations of the J-Q theory were put forth by O'Dowd and Shih $[5,6,7]$ beginning with an examination of the boundary layer model defined by the first two terms of the Williams linear elastic crack-tip solution [9]

$$
\sigma_{i j}=\frac{K}{\sqrt{2 \pi r}} f_{i j}(\theta)+T \delta_{1 i} \delta_{1 j}
$$

An evaluation of the stress state ahead of a crack in the elasticplastic material with a yield stress of $\sigma_{y^{\prime}}$ calculating the difference field by carefully examining the Small-Scale Yielding (SSY) model for various values of ' $\mathrm{T}$ ' against the SSY model for $\mathrm{T}=0$, O'Dowd and Shih determined the following relationship

$$
\left(\sigma_{i j}\right)_{\text {diff }}=\left(\sigma_{i j}\right)_{S S Y}-\left(\sigma_{i j}\right)_{S S Y, T=0}
$$

This difference resulted in a definition of $Q$ as

$$
Q=\frac{\sigma_{\theta \theta}-\left(\sigma_{\theta \theta}\right)_{S S Y: T=0}}{\sigma_{y}} \text { at } \theta=0, r=\frac{2 J}{\sigma_{y}}
$$

Where $\left(\sigma_{\theta \theta}\right)_{S S Y, T=0}$ is the SSY crack opening stress for the reference $\mathrm{T}=0$ configuration and $\sigma_{\theta \theta}$ is the opening stress ahead of an actual crack. The $Q$ value is evaluated at $2 J / \sigma_{y}$ since this value lies outside of the finite strain region but well within the bounds of the J-Q annulus defined by O’Dowd [6]. 
The Q-parameter can also be defined by considering the difference of the hydrostatic stress field $\left(\sigma_{\mathrm{h}}\right)$ between the actual crack and the reference SSY, $\mathrm{T}=0$ configuration

$$
Q=\frac{\sigma_{h}-\left(\sigma_{h}\right)_{S S Y}}{\sigma_{y}} \text { at } \theta=0, r=\frac{2 J}{\sigma_{y}}
$$

Based on the J-Q theory, values of $Q$ less than zero $(Q<0)$ signify low triaxiality ahead of the crack-tip coupled with the loss of J-dominance, while values of $Q$ greater than zero $(Q>0)$ signify high triaxiality ahead of the crack tip and agreement with the HRR solution.

\section{Numerical Results}

In Reakes, et al [2] the J-integral values for a threaded fastener were examined along various crack fronts and at multiple angular orientations, where the geometry of the crack was modeled as an elliptical crack having varying values of width (2a) and depth (b), (Figure 1). The Research of Reakes, et al. [2] is extended here by shifting focus to an evaluation of crack tip stresses. Readers are referred to Reakes, et al. [2] for detailed description of material properties and finite element models. This analysis was only done for the cases of the crack being perpendicular to the loading direction due to the difficulty in separating J for Mode I loading and J for Mode II and Mode III. The values of angular distribution of hydrostatic stress were plotted at a radius of $\mathrm{r} /\left(\mathrm{J} / \sigma_{\mathrm{y}}\right)=2$. Initial comparisons of the distribution for the fastener model against the plane strain, small-scale yielding model with $\mathrm{T}=0$ reveal a deviation in the hydrostatic stress state between the theoretical solution and the fastener finite element model.

Values of T in the SSY model are then adjusted to obtain the calculated negative $Q$ value needed to bring the triaxiality effects on the stress state in line with the calculated values in the fastener model. It can be seen from (Figure 2a) that there is a fair amount of agreeance between
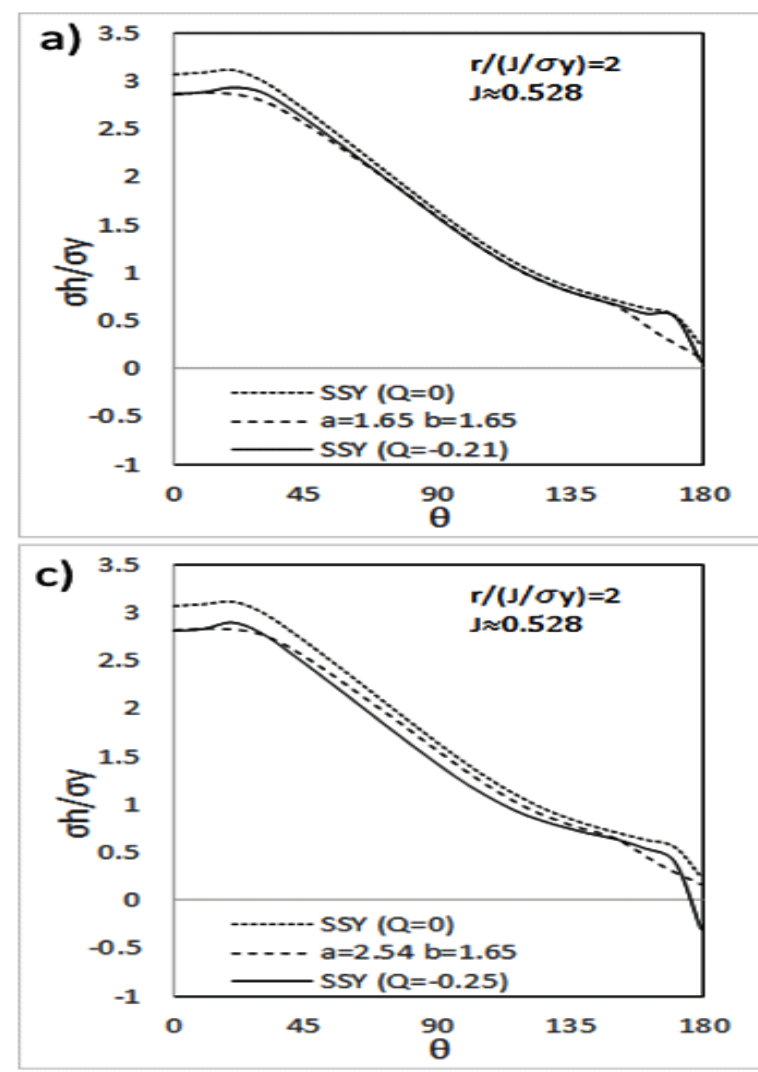

the Small-Scale Yielding [SSY] model for $\mathrm{Q}=-0.21778$ and the model for $\mathrm{a}=\mathrm{b}=1.651 \mathrm{~mm}$ model. Beyond the value of $\theta=155$ degrees the Small-Scale Yielding [SSY] results no longer represent the stress state, but since this point lies outside of the critical area for crack propagation this deviation is of little current significance to this body of work, though future investigation of this phenomenon could be of interest.

This process was then repeated for the point on $\mathrm{a}=\mathrm{b}=1.651 \mathrm{~mm}$ model at a value of $s=0$, as shown in (Figure 3a). At this point, the difference in the SSY model and the fastener model is on the scale of the yield stress. To match the angular distribution for the value of $\theta=0$ a $T / \sigma_{y}$ of 1.07 was used. A difference this large provided a less ideal curve fit when compared to the fastener model. This result was also observed by Faleskog $[10,11]$ in his comparisons for Single-Edge Notch [SEN] and specimens that were loaded in both bending and tension.

To investigate the influence of crack dimensions on hydrostatic stress state, plots were created for all crack dimensions considered in Reakes, et al. [2] of the hydrostatic stress against $\theta$ at "s" values of 0 and 0.5 . In (Figure 2) all configurations showed an increase in $Q$ at an s value of 0.5 with the greatest increase occurring for $a=1.651, b=2.54$ variant. This indicates that an increase in crack depth causes an exaggerated deviation

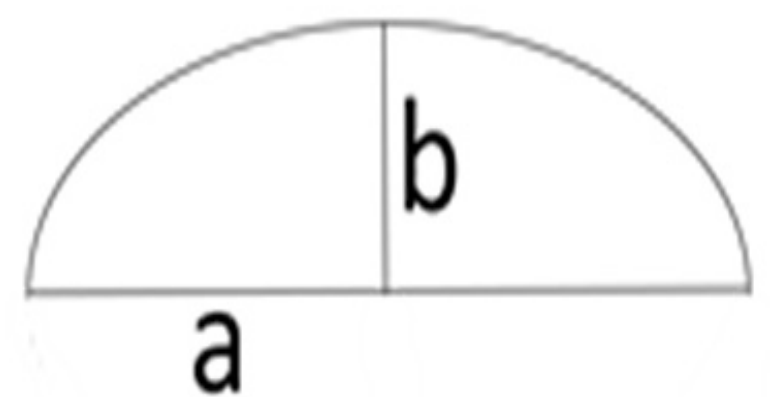

Figure 1: Definition of Control Variables for an Elliptical Crack.
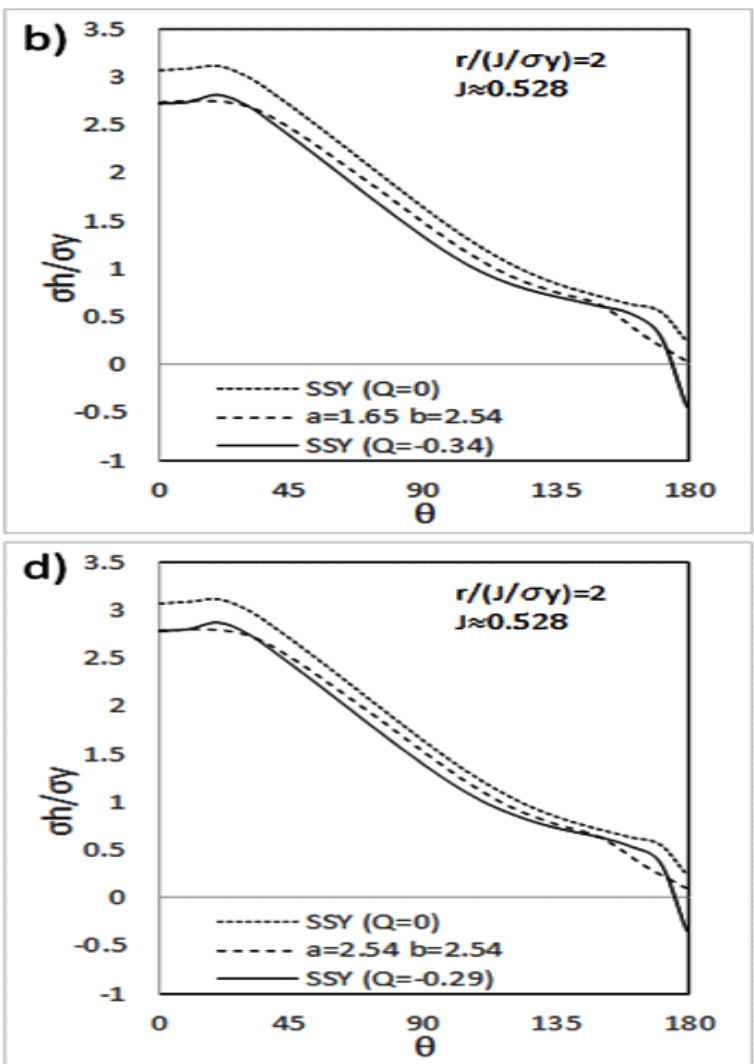

Figure 2: Angular variation at $\mathrm{r} /\left(\mathrm{J} / \sigma_{\mathrm{y}}\right)$ of the hydrostatic stress for the SSY model against 4 configurations of elliptical crack front in a threaded fastener at a position of $s=0.5$ : a) $a=b=1.651 \mathrm{~mm}$ resulting in $\mathrm{Q}=-0.21$; $\mathrm{b}$ ) $\mathrm{a}=2.54 \mathrm{~mm}, \mathrm{~b}=1.651 \mathrm{~mm}$ resulting in $\mathrm{Q}=-0.34 ; c) \mathrm{a}=1.651 \mathrm{~mm} \mathrm{~b}=2.54 \mathrm{~mm}$ resulting in $\mathrm{Q}=-0.25$; ) $\mathrm{a}=\mathrm{b}=2.54 \mathrm{~mm}$ resulting in $\mathrm{Q}=-0.29$ 


\section{High Strength Steel}

from triaxiality of the SSY model at a value of $\mathrm{T}=0$ than geometries for which the crack width is increased. An increase in both the width and depth direction appears to have an averaging influence on the value of 'Q'. However, further investigation of additional crack front geometries would be needed to verify this effect and is not presented in this paper. Stresses in the range of $\theta=35$ to $\theta=155$ reveal that deviations from the MBL solution for low levels of constraint are significant for the chosen configuration.

Considering the values of $Q$ for $s=0$ an opposite effect is seen than the one just discussed. (Figure 3 ) shows the variation of hydrostatic stress
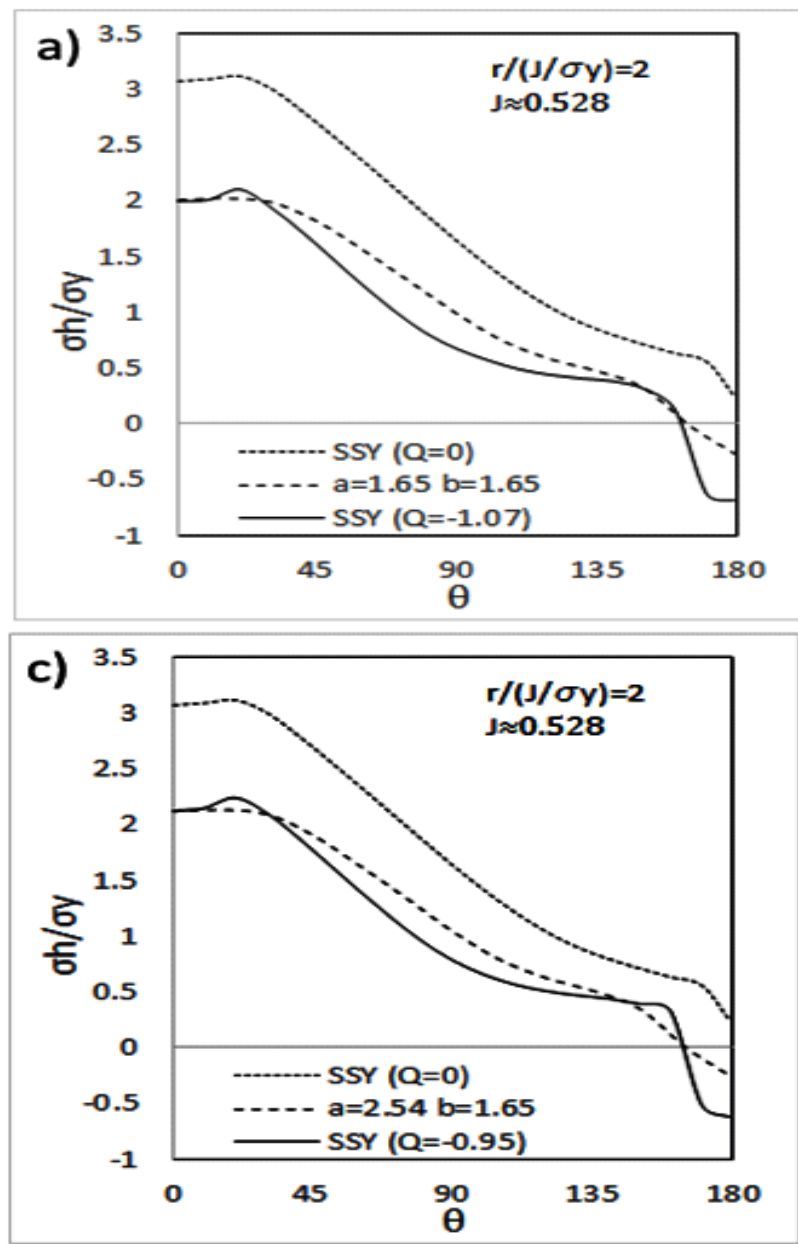

with angle $\theta$ at this location. From the results, it is seen that a high $\mathrm{Q}$ related to a stress that exceeds the yield stress for an initial configuration of $\mathrm{a}=\mathrm{b}=1.651 \mathrm{~mm}$, decreases with an increase in both crack width and depth. Changes in crack size appear to have a cumulative effect on the $Q$ parameter as the crack size progressively increases. This causes a decrease in the hydrostatic stress. At highly negative levels of the parameter $Q$ a deviation in the hydrostatic stresses of the SSY model from the fastener model is observed between $\theta \approx 35$ and $\theta \approx 155$ for all cases. It is observed that this difference decreases as $Q \rightarrow 0$. These results bring into question as to whether a third-parameter may be needed to properly define the stress state for the chosen configuration of the crack.
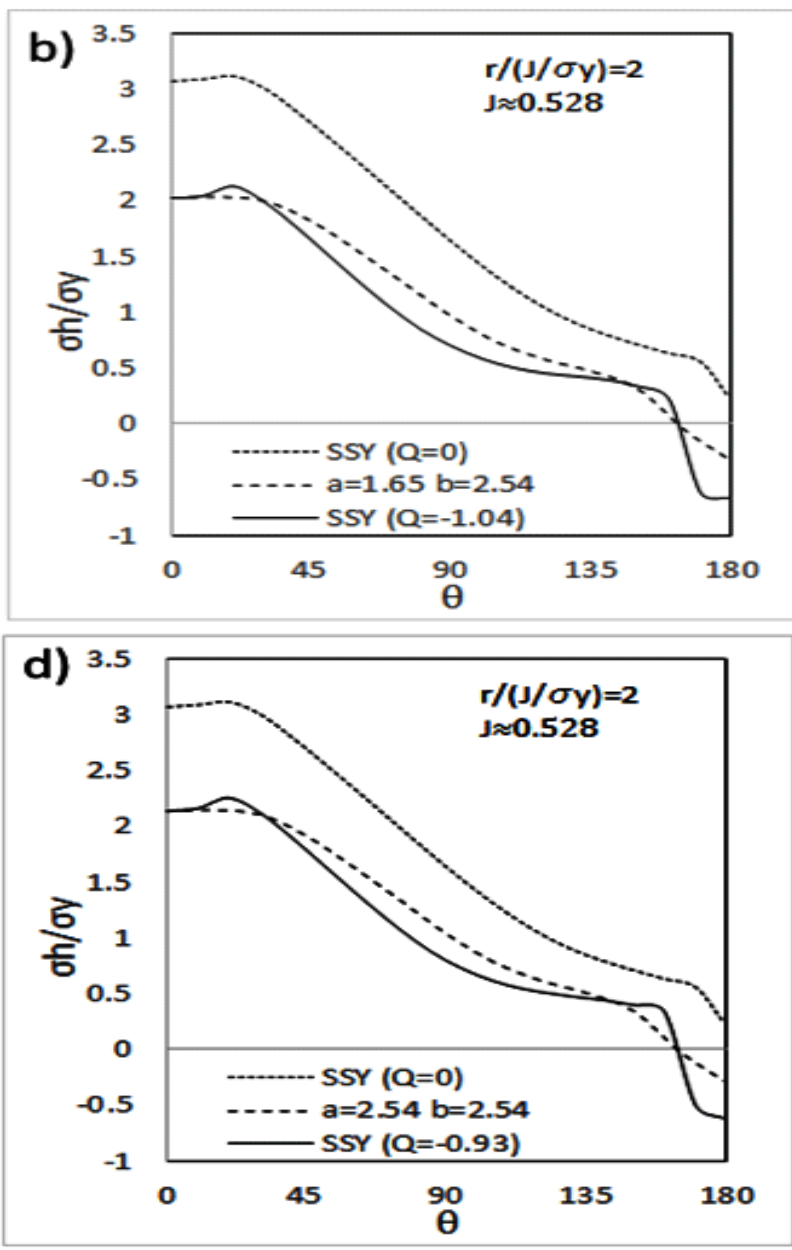

Figure 3: Angular variation at $\mathrm{r} /\left(\mathrm{J} / \sigma_{\mathrm{y}}\right)$ of the hydrostatic stress for the SSY model against 4 configurations of elliptical crack front in a threaded fastener at a position of $\mathrm{s}=0$ : a) $\mathrm{a}=\mathrm{b}=1.651 \mathrm{~mm}$ resulting in $\mathrm{Q}=-1.07 ; \mathrm{b}$ ) $\mathrm{a}=2.54 \mathrm{~mm}, \mathrm{~b}=1.651 \mathrm{~mm}$ resulting in $\mathrm{Q}=-1.04 ; \mathrm{c}$ ) $\mathrm{a}=1.651 \mathrm{~mm} \mathrm{~b}=2.54 \mathrm{~mm}$ resulting in $\mathrm{Q}=-0.95 ; \mathrm{d}$ ) $\mathrm{a}=\mathrm{b}=2.54 \mathrm{~mm}$ resulting in $\mathrm{Q}=-0.93$

Seeing such a large variation in the value of $Q$ at the free edges of the crack front and the crack root, the values of $Q$ along the crack front were plotted alongside with the values of J along the crack front to bring to light the necessity of the two-parameter approach. Single parameter fracture mechanics would predict crack propagation at the area in (Figure 4) where J is the highest. It can be seen from this figure, that presence of low triaxiality in this region defines the value of $Q$ to be small when compared to value at the crack front center. Therefore, it is expected from the J-Q Locus that crack propagation has the potential to occur in any region where a given range of values for $\mathrm{J}_{\mathrm{c}}$ and $\mathrm{Q}$ intersect.

Figure 5 shows how $J$ and $Q$ vary as the load is increased. It can be seen from the chart that the J parameter increases with additional loading displacement while the parameter $Q$ becomes increasing negative as the low-constraint effects grow. This implies that as the loading progresses farther into the nonlinear range the application of the two-parameter J-Q approach to fracture mechanics becomes necessary to accurately predict

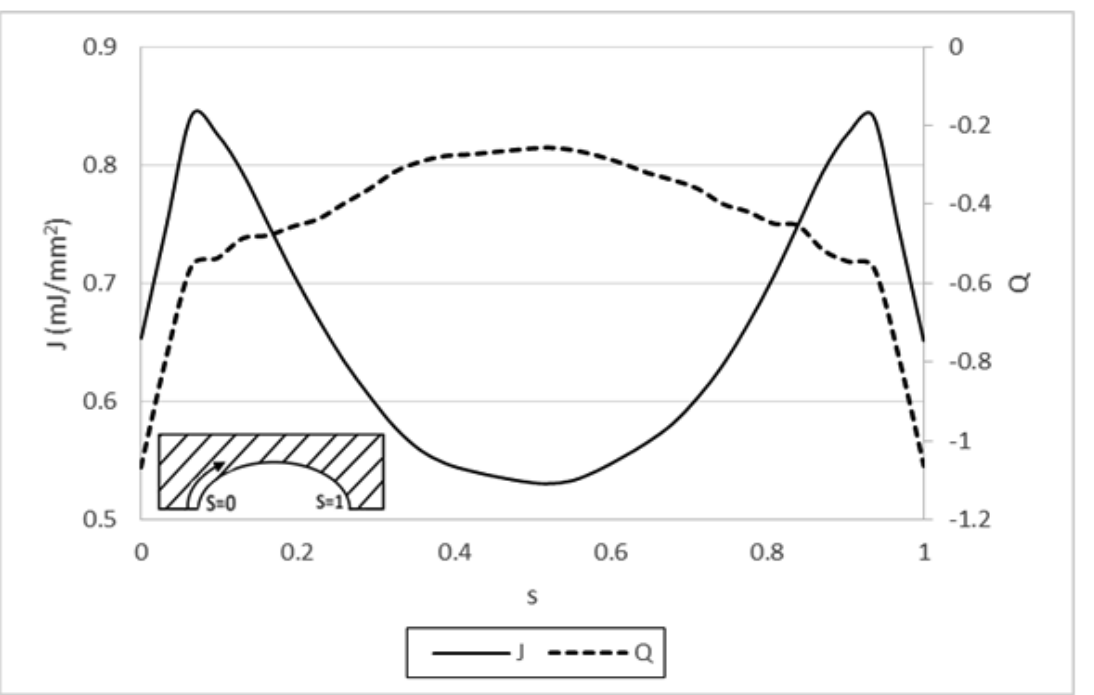

Figure 4: Variation of $\mathrm{J}$ and $\mathrm{Q}$ along the crack front for a specimen flaw of dimensions $\mathrm{a}=\mathrm{b}=1.651 \mathrm{~mm}$ demonstrating the need for a two parameter fracture mechanics approach to failure analysis. 


\section{High Strength Steel}

failure. In the early stages of the loading parameter $Q$ is in the positive range indicating that a single parameter J theory can be used within this region since the failure will be J-dominated. As parameter Q progresses in the negative region, the stresses predicted by the J-integral method will exceed the actual stresses seen in the component providing indication for the crack to not extend in a manner as predicted using the single parameter J method.

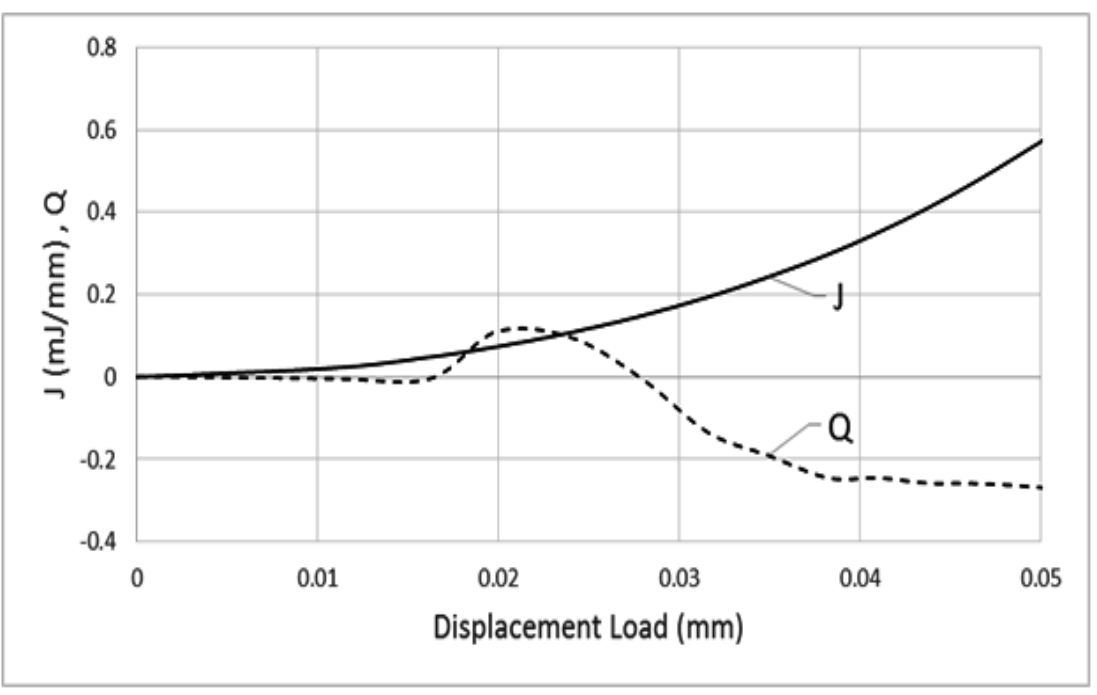

Figure 5: Variation of parameters J and Q with displacement load perpendicular to the crack plane.
The next course of this investigation was to examine the opening stress and radial stress ahead of the crack tip to verify the two-parameter J-Q approach. From (Figure 6), which represents a crack having a geometry of $a=b=1.651 \mathrm{~mm}$ compared to values of the MBL model with a parameter $Q$ set to -0.207 , it is noted that the crack opening stress has excellent agreement for a value of $\mathrm{r} /\left(\mathrm{J} / \sigma_{\mathrm{y}}\right)=2$. This is to be expected since this is the point at which $Q$ was calculated. Beyond a $\mathrm{r} /\left(\mathrm{J} / \sigma_{\mathrm{y}}\right)$ value of 2 the Small-Scale Yielding (SSY) curve continues to hold a close fit to the fastener model. By examining the radial stress, slightly less agreement between the Small-Scale Yielding (SSY) model and the fastener model is seen. This implies that the third term may have a greater influence on overall hydrostatic stress than the two-parameter J-Q theory accounts for configurations of his type.

Examination of the opening and radial stresses for the 3 configurations tested, shown in (Figure 6), reveals similar results to $a=b=1.651$ model. There is a close fit for all cases of the opening stress and the radial stress. As the parameter, $\mathrm{Q}$ is increased it is seen that both the opening and radial stress for the Small-Scale Yielding (SSY) model are shifted below the curves extracted from the fastener models. These shifts indicate that the SSY model for values of the $Q$ parameter below -0.21 do not predict the contributions from the out-of-plane stress.
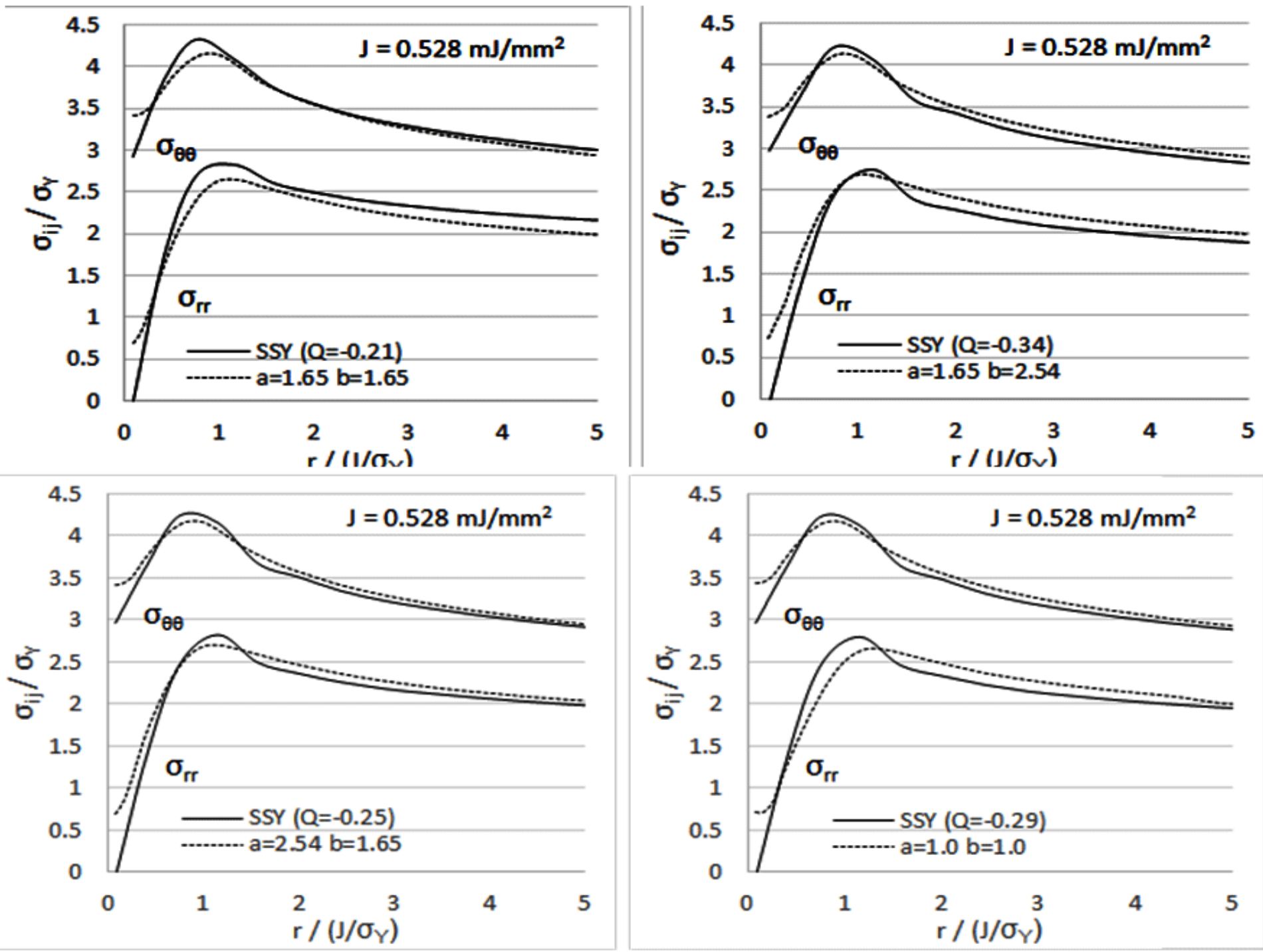

Figure 6: Stress distribution ahead of the crack tip at $\theta=0$ plotted against $\left(r / J \sigma_{\mathrm{y}}\right)$ for crack opening stress $\left(\sigma_{\theta \theta}\right)$ and radial stress $\left(\sigma_{\mathrm{rr}}\right)$ for: $\left.\mathrm{a}\right) \mathrm{a}=\mathrm{b}=1.651 \mathrm{~mm}$, SSY at $\mathrm{Q}=0.21$; b) $\mathrm{a}=2.54 \mathrm{~mm}, \mathrm{~b}=1.651 \mathrm{~mm}$, SSY at $\mathrm{Q}=-0.34 ; \mathrm{c}$ ) $\mathrm{a}=1.651 \mathrm{~mm} \mathrm{~b}=2.54 \mathrm{~mm}$, SSY at $\mathrm{Q}=-0.25 ; \mathrm{d}) \mathrm{a}=\mathrm{b}=2.54 \mathrm{~mm}, \mathrm{SSY}$ at $\mathrm{Q}=-0.2$ 


\section{Conclusions}

Based on a numerical study of the stress state in the crack front region of a threaded fastener, made from high strength steel, following are the key findings

1. The single-parameter fracture mechanics theory characterized by the J-integral cannot describe the stress field of the surface- Cracked fastener geometries. The actual stress field deviates from the Hutchinson-RiceRosengren (HRR) solution.

2. Effects of triaxiality in the low-constraint region can be approximated by using the two-parameter J-Q theory proposed by O'Dowd and Shih. This method helps in bringing the hydrostatic stress state ahead of the crack front for the case of Modified Boundary Layer solution in tune with the numerical solution for a threaded fastener.

3. Analysis reveals that the J-integral along the crack front to have its highest value near the free edges, while the value of $Q$ has its highest value at the root of the crack. From this it can be inferred that a single parameter fracture mechanics approach may not be able to predict the location of failure.

4. It is shown that that magnitude of the parameter $\mathrm{Q}$ becomes increasingly negative as the loading progresses into the large strain region and the stress field deviates from the HRR field.

\section{References}

1. Ramachandran V. Failure Analysis of Engineering Structures: Methodology and Case Histories. Materials Park, OH: ASM International. 2005;102-106:130-132.

2. Reakes C, Gao X, Srivatsan TS. Analysis of Threaded Fasteners of a High Strength Steel: Role of Flaw Size and Orientation - A Numerical Study Neural, Parallel and Scientific Computations. 2016;24:335-350.

3. Hutchinson JW. Singular Behavior at the End of a Tensile Crack in a Hardening Material. Journal of the Mechanics and Physics of Solids. 1968;16(1):13-31.

4. Rice JR, Rosengren GF. Plane Strain Deformation near a Crack Tip in a Power-law Hardening Material. Journal of the Mechanics and Physics of Solids. 1968;16(1):1-12.

5. O'Dowd NP, Shih CF. Family of Crack-tip Fields Characterized by a Triaxiality Parameter-I. Structure of Fields. Journal of the Mechanics and Physics of Solids. 1991;39(8):989-1015.

6. O’Dowd NP, Shih CF. Two-parameter Fracture Mechanics: Theory and Applications. Fracture Mechanics. 1994;24:21-47.

7. O’Dowd NP, Shih CF. Family of Crack-tip Fields Characterized by a Triaxiality Parameter-II. Fracture Applications. Journal of the Mechanics and Physics of Solids. 1992;40(5):939-963.

8. Henry BS, Luxmoore AR. The Stress Triaxiality Constraint and the Q-value as a Ductile Fracture Parameter. Engineering Fracture Mechanics. 1997;57(4):375-390.

9. Williams ML. On the Stress Distribution at the Base of a Stationary Crack. Journal of Applied Mechanics.1957;24(1):109-114.

10.Faleskog J, Zaremba K, Nilsson F, Oberg H. An Investigation of Two- and ThreeDimensional Elasto-Plastic Crack Growth Experiment. Defect Assessment in Components - Fundamentals and Applications, ESIS/EGF9. 1991;333-343.

11.Faleskog J, Nordlund P. Near-tip Field Characterization and J-integral Evaluation for Nonproportional Loads. International Journal of Solids and Structures. 1994;31(1):1- 